\title{
Wearable Technology: Healthcare Product Design For Participation Of Tetraplegics In Society
}

\section{SIGRADI2018 TECHNOPOLITICAS \\ xxii congresso da sociedade iberoamericana de gráfica digital 22th conference of the iberoamerican society of digital graphics 07|08|09|novembro|2018 iau usp | são carlos | sp br}

\author{
Anelise Ventura \\ EESC USP | Brazil | aneliseventura@usp.br \\ Renato Varoto \\ FCM UNICAMP | Brazil | rvaroto@yahoo.com.br
}

Alberto Cliquet Junior

EESC USP | Brazil | cliquet@usp.br

\begin{abstract}
This paper discusses technological and theoretical issues that conform the product design proposal called technological wearable: a system that integrates body and equipment for physical rehabilitation of quadriplegic patients and their participation into society. The relevance of the work is to enhance the malleability and performance efficiency of the current system, through product design with complex surfaces, in addition to its customization and optimization, with the implementation of an open source plug-in, reducing steps and inaccuracies, to give more autonomy to the patient and to generate discussions in the areas that can use the same process.
\end{abstract}

Keywords: Wearable; Healthcare Product Design; Tetraplegia; Complex Surfaces; Optimization

\section{INTRODUÇÃO}

A lesão medular é um dano grave e no Brasil acomete cerca de mais de 10 mil novos casos por ano, segundo as diretrizes do Ministério da Saúde de 2015, com repercussão física, psíquica e social. Limitações motoras e sensoriais são algumas das consequências da lesão que afetam membros superiores de lesados medulares tetraplégicos, dificultando a realização de atividades da vida diária, como por exemplo levar um copo de água à boca e a sua participação da sociedade.

A lesão medular pode ser traumática, devido acidente de carro, ferimentos por arma de fogo e impacto por atividade esportiva ou recreativa. E não traumática, resultante de doenças ou patologias, como trombose, embolia, hérnia de disco, esclerose múltipla, e tumores que comprimem a medula. (Eng; Miller, 2006). A paralisia em diferentes níveis e partes do corpo, bem como alterações na sensibilidade e comorbidades (Eng; Miller, 2006), são as consequências da lesão medular, que está dividida em tetraplegia e paraplegia. Esta pesquisa está restrita ao desenvolvimento de produto aos membros superiores de pacientes tetraplégicos, os quais têm comprometidos os movimentos de alcance com as mãos, preensão dos objetos e coordenação em relação à posição e orientação dos membros no espaço (Varoto; Ferreira; Alonso; Beinotti e Cliquet, 2011).

Atualmente, técnicas de reabilitação artificial, via Estimulação Elétrica Neuromuscular (EENM) em membros superiores, têm respondido de modo promissor em indivíduos tetraplégicos, para que aqueles movimentos sejam estimulados e reaprendidos, tornando os lesados medulares tetraplégicos menos dependentes
(Varoto e Cliquet, 2015). A EENM é aplicada por meio de eletrodos de superfície, os quais são diretamente fixados no corpo de pacientes com acessórios e materiais usuais de eletrônica (Figura 1) e seu desempenho é fundamental para o bom funcionamento dos estímulos de grupos musculares, sem expectativas, a princípio, das funções neurológicas voltarem ao normal, mas sim de ser um meio para a restauração artificial muscular e motora (Peckham; Keith; Freehafer, 1988).

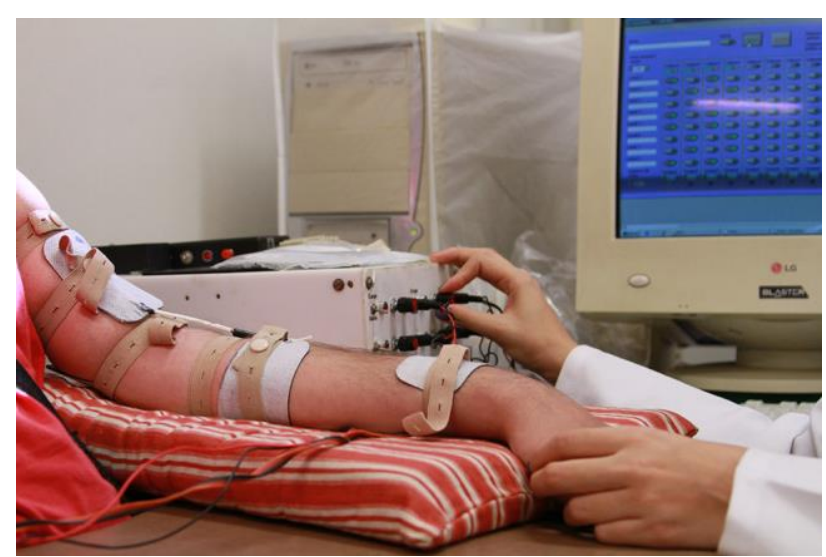

Figura 1. Reabilitação funcional motora por Estimulação Elétrica Neuromuscular. Fonte: Adaptado de Joon Ho Kim, 2011

Alguns exemplos de produtos e projetos desenvolvidos para o tratamento com o uso da EENM e que possivelmente tenham alguma aproximação com 0 vestível tecnológico a ser desenvolvido, podem ser aqui mencionados. 


\section{NESS H200 ${ }^{\circledR}$ - HANDMASTER}

O NESS H200 ${ }^{\circledR}$ - Handmaster (Figura 2) desenvolvido por Roger Nathan e seu grupo de pesquisa na Universidade de Israel é composto por três eletrodos de superfície e uma tala para o antebraço. O sistema atua por meio de EENM acionado pelo paciente. Testes foram realizados em dez pacientes tetraplégicos. Em termos desempenho o produto apresentou benefícios funcionais aos pacientes. Em termos do design do produto ele não se apresentou ergonômico para todos os tamanhos de antebraços testados, sendo que dentre dez unidades do produto, três não serviram em pacientes, devido a tala ser muito pequena (Snoek et al., 2000)

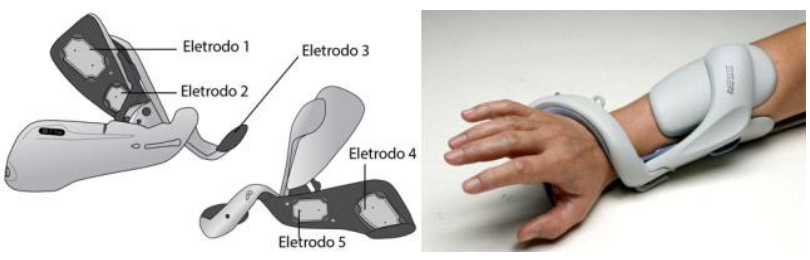

Figura 2. NESS $\mathrm{H}_{2} 00^{\circledR}$ - Handmaster Fonte: Adaptado de Snoek et al., 2000.

\section{CARPAL SKIN}

O Carpal Skin (Figura 3) foi desenvolvido pela designer e pesquisadora do Instituto de Tecnologia de Massachusetts (MIT) Neri Oxman, pacientes com a síndrome do túnel do carpo. O vestível tem como objetivo minimizar as dores provenientes da patologia, mapeandoas de acordo com a intensidade e a duração e, a partir disso, construir um produto que dialogue com a dor, por meio da distribuição de materiais rígidos e macios de acordo com a anatomia e fisiologia de cada paciente. A complexa relação entre parâmetros de engenharia, design e requisitos fisiológicos nortearam 0 desenvolvimento do Carpal Skin (Oxman, 2010).
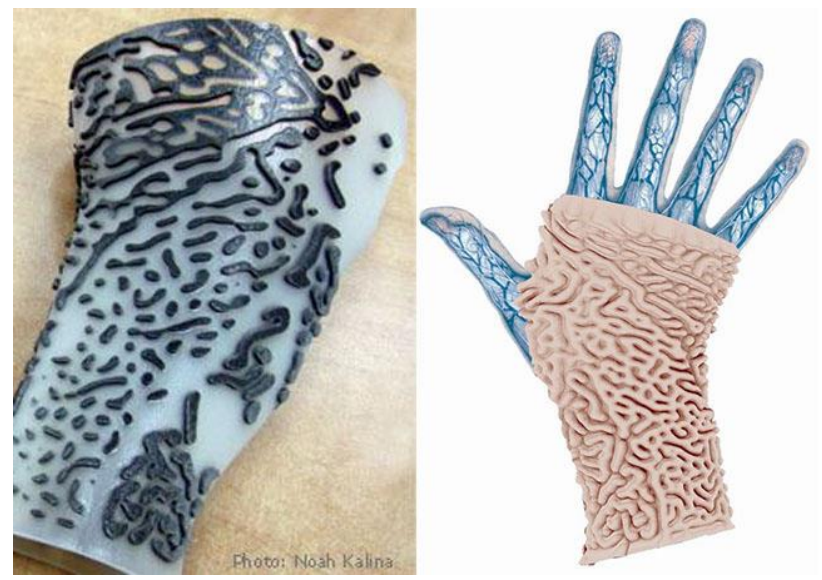

Figura 3. Carpal Skin Fonte: Adaptado de https://sap.mit.edu/article/standard/introducing-two-new-faculty e Oxman, 2010.

\section{ESTIMULADOR ELÉTRICO FUNCIONAL INTELIGENTE (INTFES)}

O Estimulador Elétrico Funcional Inteligente (IntFES) (Figura 4), foi desenvolvido por um grupo de pesquisadores da Universidade de Ljubljana, Eslovênia para ser aplicado em membros superiores de pacientes com AVC. O projeto consiste no desenvolvimento de um sistema composto por uma matriz de eletrodos, que podem ser ativados individualmente, tornando-o mais seletivo em relação aos grupos musculares e produzindo movimento funcional. Este sistema pode ser aplicado em ambientes clínico e domiciliar. Três pacientes usaram este dispositivo e chegou-se à conclusão de que cada paciente tem que ter sua matriz de eletrodos multi-pad de acordo com a anatomia do antebraço. Além disso, o sistema melhorou o problema do tempo de colocação dos eletrodos e a sua deterioração devido ao uso em relação aos eletrodos comercialmente disponíveis (Malešević, 2012).

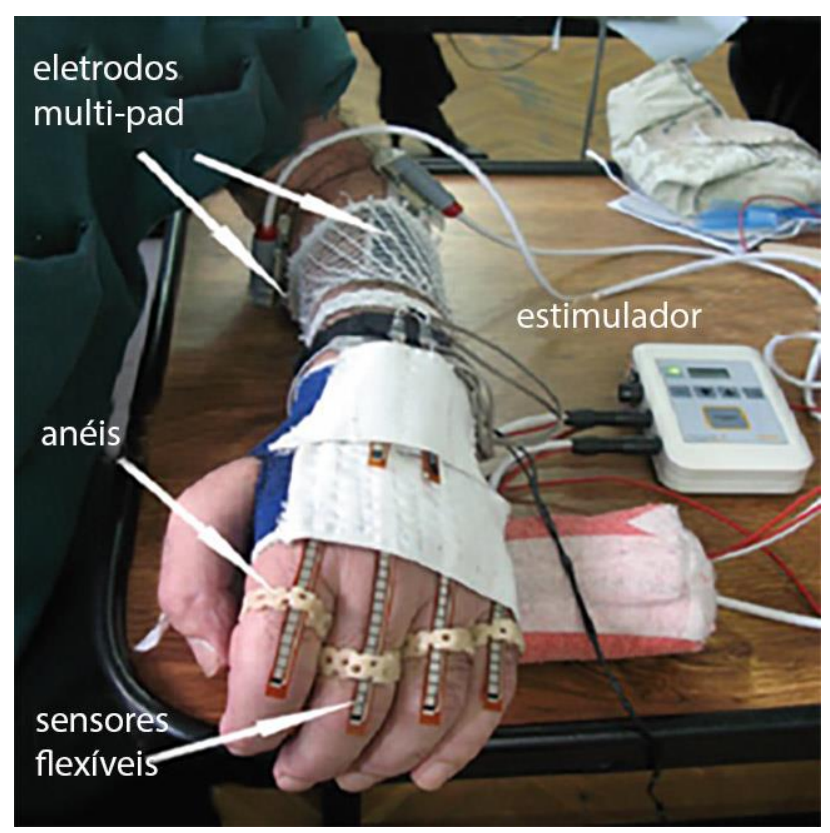

Figura 4. Estimulador Elétrico Funcional Inteligente (IntFES). Fonte: Adaptado de https://jneuroengrehab.biomedcentral.com /track/pdf/10.1186/1743-0003-9-66

Os exemplos de vestíveis apresentados caminham em direção à otimização, performance e integração do sistema com o usuário. Importantes análises críticas sobre estes conceitos foram levadas em consideração para a elaboração do vestível proposto. A otimização, por exemplo, contribuirá para que o sistema seja mais ágil e prático em relação à colocação do vestível e ao posicionamento fixo dos eletrodos, além de melhorar a performance do sistema como um todo. A integração com o usuário, ergonomia e design formal dos produtos demonstraram que são importantes fatores para que diferentes indivíduos possam usá-los, atendendo a variações antropométricas e de maneira que seja prático de ser colocado e retirado, uma vez que pacientes tetraplégicos possuem os membros superiores sem mobilidade.

Assim, a retomada dos movimentos naturais do corpo humano é o conceito que norteia este projeto e está diretamente ligado à ideia de integração e personalização. A relevância do trabalho é potencializar a capacidade de maleabilidade e eficiência performática do vestível tecnológico de superfícies complexas, com a integração do membro superior ao conjunto de eletrodos, de modo a dar mais liberdade de movimento ao paciente e aos profissionais da saúde realizarem suas atividades. 
Além disso, transformar as relações de uso e percepções visual e tátil, proporcionando diferentes relações com o usuário.

O objetivo principal deste trabalho é a exploração projetual, para uma possível independência e participação de indivíduos portadores de lesão medular, em específico tetraplégicos, na sociedade. Pretende-se, com esta produção, aprimorar o processo de reabilitação dos membros superiores dos lesados medulares, contribuindo com os pacientes e equipe diretamente envolvida composta por médicos, fisioterapeutas, terapeutas ocupacionais, engenheiros e designers e, simultaneamente, investigar as possibilidades de torná-la uma plataforma de comunicação colaborativa que sirva de meio a processos de participação eficazes entre pacientes e equipe e pacientes com a sociedade. $O$ objetivo específico do trabalho é personalizar sistema de reabilitação por EENM atual, tornando-o único para cada paciente e otimizar suas etapas, reduzindo suas imprecisões.

Portanto, procura-se discutir o design de um produto que funcione como um sistema integrador do usuário, com os elementos de eletrônica e equipamentos que o compõem, denominado vestível tecnológico. No caso abordado serão descritos e analisados os possíveis dados de entrada em que é estruturado o produto para que possa receber alterações que viabilizem e potencializem o seu uso.

\section{METODOLOGIA}

O projeto do vestível tecnológico está em andamento e depende de um conjunto de fatores para a sua boa execução. Os elementos que compõem a metodologia são discutidos a seguir.

\section{AVALIAÇÃO CLÍNICA}

O vestível será desenvolvido para pacientes do Laboratório de uma Universidade do Brasil, lesados medulares tetraplégicos, de ambos os sexos, composto por 5 voluntários, com idade entre 20 e 65 anos e dentre eles um paratleta, com tempo de ocorrência da lesão superior a 1 ano. A escolha destes voluntários deve-se às características variadas de gênero do público e suas distinções antropométricas.

Critérios de exclusão são determinados na presença de déficit cognitivo, presença de lesões na pele dos membros superiores, incapacidade de manter-se sentado livremente em cadeira de rodas e com suporte nas costas, que apresente déficits neurológicos prévios, cirurgia de transferência de tendões envolvendo membros superiores e distúrbios osteomusculares no membro superior.

\section{CARÁTER INTERDISCIPLINAR}

O desenvolvimento de produtos na área da saúde, sobretudo aqueles que se utilizam de dispositivos elétricos e eletrônicos, como é o caso do vestível tecnológico apresentado neste trabalho, envolve uma equipe multidisciplinar como médicos, fisioterapeutas, engenheiros e designers, e que trabalhe de forma interdisciplinar (Romero, 2018). Além disso, os usuários envolvidos exercem funções distintas, como é o caso do manuseio do produto pela equipe técnica e do uso pelos pacientes tetraplégicos. Somado a isso, tem-se as características antropométricas muito distintas e deformidades dos membros superiores resultantes da lesão que variam para cada paciente, o que resulta na necessidade de produtos personalizados. Isto implica em um grande número de complexidades que ocorre no momento da concepção e do desenvolvimento do design do produto. Um fluxograma foi desenvolvido para a compreensão do processo interdisciplinar que abrange o universo do desenvolvimento do vestível tecnológico (Figura 5).

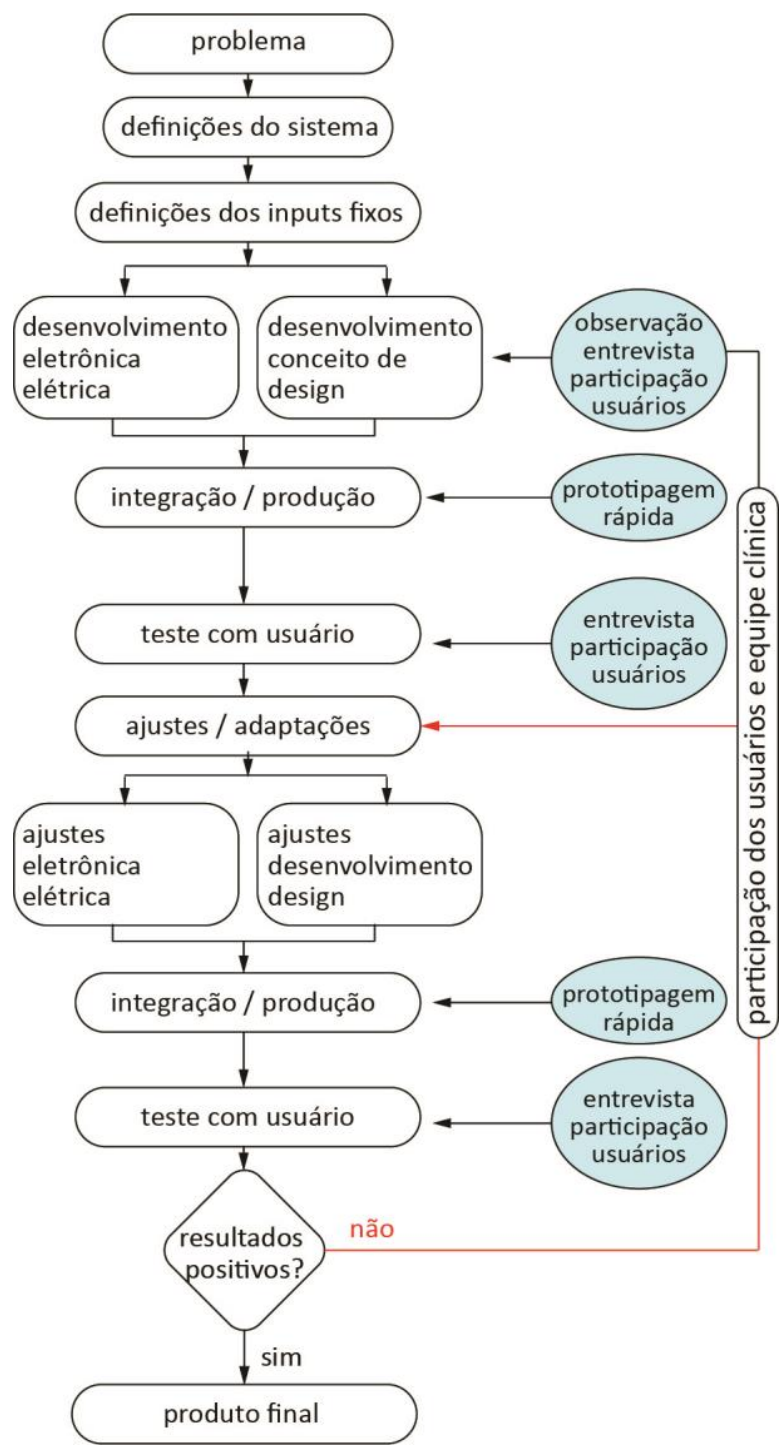

Figura 5. Fluxograma de processo de design interdisciplinar proposto para o desenvolvimento do vestível tecnológico. Fonte: Autoria própria, adaptado de Romero, 2010

\section{REVISÃO E APRIMORAMENTO DO MODO DE APLICAÇÃO DA EENM}

Atualmente diferentes técnicas de EENM têm sido aplicadas para a reabilitação de membros superiores. Um protocolo de reabilitação com o uso da EENM associada a movimentos funcionais e repetitivos, caracterizado por oito canais de estimulação e oito eletrodos de superfície, com posições e locais específicos (Figura 6), foi desenvolvido pelos grupos de pesquisa do Laboratório de 
Biocibernética e Engenharia de Reabilitação [LABCIBER], do Departamento de Engenharia Elétrica e de Computação, da Escola de Engenharia de São Carlos [EESC], da Universidade de São Paulo [USP] e do Laboratório de Reabilitação do Aparelho Locomotor [LABRAL], do Departamento de Ortopedia da Faculdade de Ciências Médicas da Universidade Estadual de Campinas [UNICAMP]. Este protocolo tem sido aplicado em membros superiores de pacientes tetraplégicos do LABRAL, por equipe técnica habilitada com resultado de reabilitação eficaz (Alonso, 2015). Esta norma é adotada no desenvolvimento do design do produto proposto.

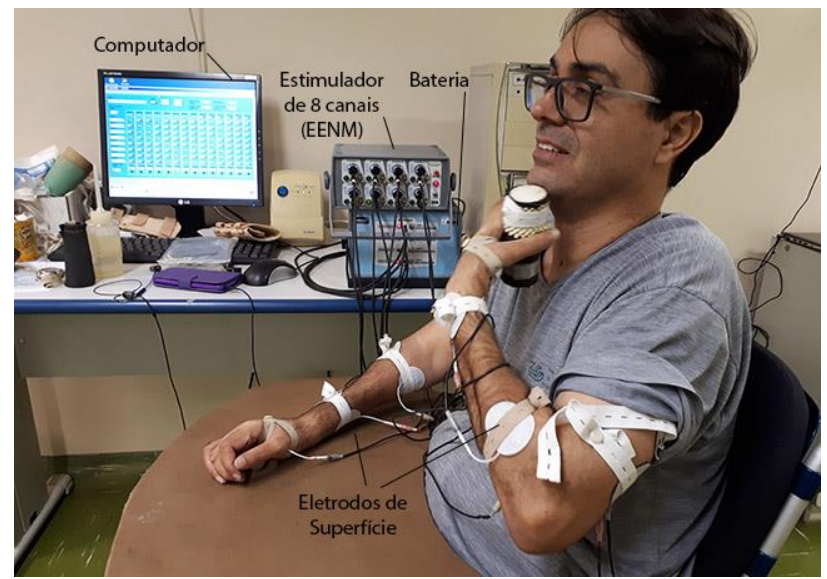

Figura 6. Equipamentos necessários para a reabilitação com EENM - computador, estimulador de 8 canais, bateria e eletrodos de superfície. Fonte: Autoria própria, 2018

Para a concepção do vestível fez-se uma análise dos componentes de eletrônica usuais na EENM. Tem-se que o estimulador de 8 canais a princípio, não pode ser alterado em sua forma, dimensão e modo de uso e a bateria, que alimenta todo o sistema, apresenta-se superdimensionada podendo ser aprimorada.

Os eletrodos de superfície (Figura 7), sensores táteis e sensores de movimento, atualmente todos são comerciais, devem ser incorporados ao vestível devido algumas deficiências descritas adiante.
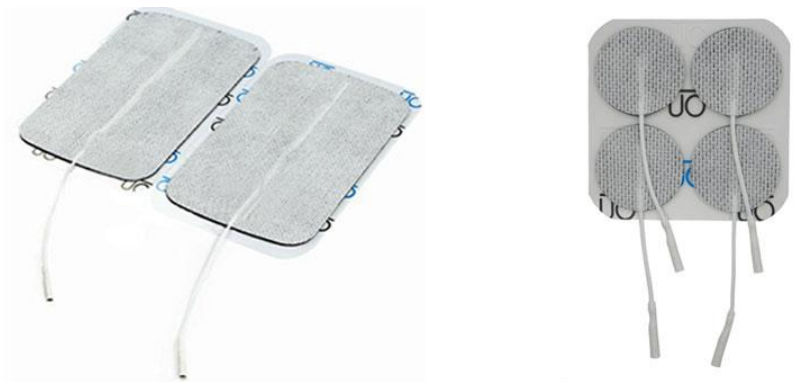

Figura 7. Eletrodos autoadesivos Valutrode ${ }^{\circledR}$ tamanhos $5 \times 9 \mathrm{~cm}$ e $3 \mathrm{~cm}$ de diâmetro respectivamente. Fonte: Adaptado de https://www.fisiofernandes.com.br/eletroterapia. Acesso em 22 outubro de 2017.

Os sensores táteis (Figura 8) são usados para identificar a preensão de objetos e possuem a capacidade de detecção de força em duas superfícies.

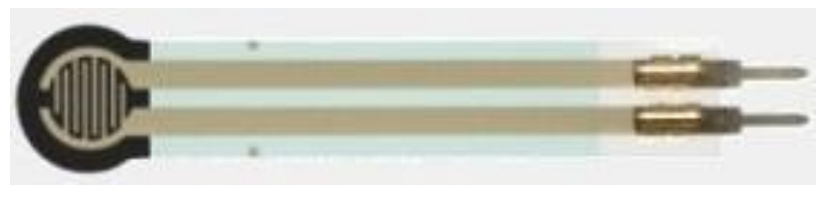

Figura 8. Sensor tátil 400 FRS Interlink®. Fonte: Adaptado de https://www.interlinkelectronics.com/fsr-400. Acesso em 22 outubro de 2017

Os marcadores de movimento regularmente usados são envolvidos em uma fita que reflete luz infravermelha e fixados no membro superior do paciente, para avaliação da eficiência e performance dos movimentos durante a reabilitação.

Neste momento, análises e revisões relacionadas à aplicação e eficiência do procedimento de reabilitação por meio da EENM têm sido feitas em conjunto com engenheiros e designers, baseado em observação e entrevista com equipe técnica e pacientes in loco. Detectou-se que o sistema de EENM atual apresenta algumas desvantagens, sendo elas: o tamanho da bateria; a colocação manual dos eletrodos sobre o membro do paciente retardando o processo; a repetição exata da posição e local de cada eletrodo sobre grupos musculares, resultando em um processo longo e, por vezes, impreciso; a perda de aderência dos eletrodos ao longo do tratamento, tornando inadequadas suas funções, de modo que alternativas paliativas sejam adotadas e a quantidade de fios que, em muitas vezes, obstruem a fluência do movimento.

Para que o sistema se torne integrado ao vestível, uma vez que este é o objetivo do projeto, sugere-se que cada parte que o compõe deva estar o mais incorporado possível ao vestível, fazendo com que estratégias de redução do tamanho e otimização dos componentes eletrônicos, como computador, bateria, fios e cabos sejam adotadas. Para o computador desktop um novo sistema foi elaborado, com o objetivo de compactá-lo e torná-lo mais amigável. Este sistema é composto por um controlador para o estimulador neuromuscular baseado em uma Raspberry Pi e uma tela touchscreen (Parente, 2017)

Quanto a bateria, um novo circuito eletrônico foi proposto para o estimulador. Como consequência (ainda não testado em bancada) as baterias foram reduzidas, já que o novo circuito é - aparentemente - mais eficiente.

\section{PROCESSO DE PROJETO BOTTOM-UP}

O processo de design paramétrico é produzido por um conjunto de regras cujas propriedades são governadas ou articuladas pelos valores de determinadas variáveis ou parâmetros. A alteração de um parâmetro acarreta na alteração dos demais, conjuntamente, de maneira que o sistema se auto organiza. Esse processo apoia-se no pensamento algorítmico, que orienta a definição destas relações entre os parâmetros, com uma sequência finita e precisa de passos (Woodburry, 2010). Esta alternativa promove combinações de parâmetros específicos e a geração de diversas relações e possibilidades no universo da geometria complexa, caracterizando-o como um processo de baixo para cima (bottom-up) (Studer et al., 1998). 
O processo de projeto paramétrico adotado para o desenvolvimento do vestível pode gerar de qualidades (outputs) ainda não exploradas, motivadas pelo comportamento dos movimentos dos membros superiores estimulados artificialmente. Isto ocorrerá devido a própria concepção de projeto adotada (bottom-up) em que todos os integrantes da equipe e pacientes participam do processo de criação desde o seu início, além dos membros superiores dos pacientes, componentes eletrônicos e performance do material adotado ao vestível fazerem parte dos dados de entrada iniciais (inputs), em contrapartida à uma readequação formal e estética do sistema existente (processo top-down).

Para que o vestível funcione como um invólucro corpóreo, é importante que ele seja modelado sobre o membro superior dos 5 voluntários. Para tal o escaneamento tridimensional de cada membro de cada paciente se faz necessário. Posteriormente, sobre o resultado deste escaneamento, os vestíveis serão modelados. A princípio, diagramas matemáticos em sistemas generativos automatizados são adotados como exploração projetual. O Diagrama de Voronoi (Figura 9), no âmbito da tecnologia computacional, surge como uma possibilidade de modelo matemático para 0 desenvolvimento da superfície do vestível. Este diagrama permite a geração de mudanças automáticas na sua forma pela inserção ou exclusão de pontos estrategicamente localizados no espaço, promovendo desdobramentos e ajustes controlados (Galvão, 2003). Essas complexidades permitem que a geometria inicial aninhe outra, proporcionando uma interação conjunta.

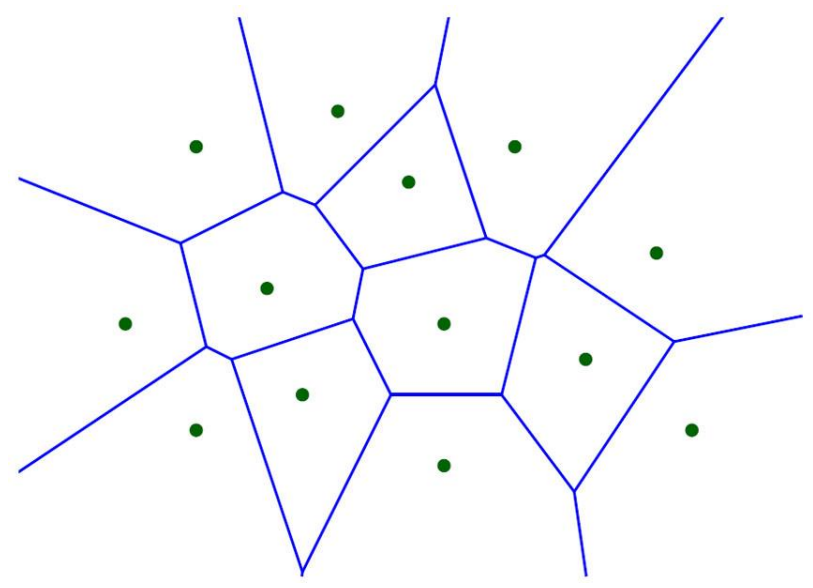

Figura 9. Diagrama de Voronoi. Fonte: Emiris; Fisikopoulos (2009).

O diagrama de Voronoi se justifica como possível modelo matemático para o desenvolvimento da superfície complexa do vestível pois os inputs fixos inseridos em termos de dimensão e posição (eletrodos, sensores, cabos e fios) deverão dialogar com os inputs que variam (dimensão e forma do braço e antebraço de cada paciente). Além disso, propõe-se um vestível com parte da sua superfície vazada (Figura 10), para tornar o produto mais leve e arejado. Os vazios também se auto organizarão de acordo com os inputs fixos e variáveis.

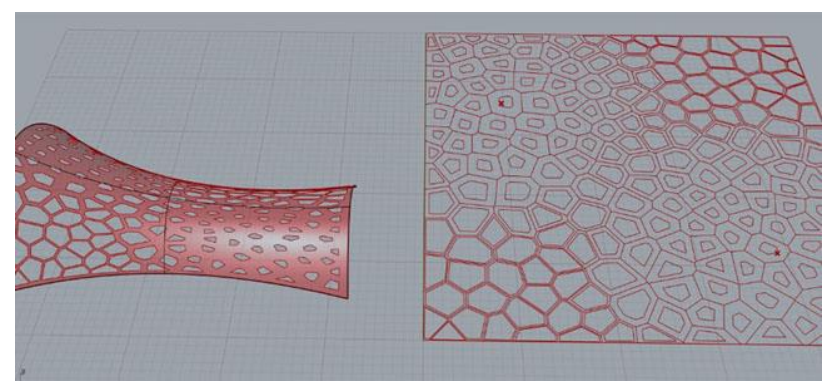

Figura 10. Diagrama de Voronoi modelado tridimensionalmente. Fonte: Adaptado de http://atlv.org/education/grasshopper/. Acesso em 10 de Março de 2018

O processo de design paramétrico com o uso do software de modelação tridimensional (Rhinoceros), em conjunto com um plug-in de algoritmo generativo (Grasshopper) é adotado como forma de investigação projetual. Suas características promovem otimização, geração de diversas possibilidades e análise simultânea de dados. Além de software para análises de elementos finitos (ainda não definido) como forma de análise de possíveis deformações e desgastes do material. Este conjunto de software é necessário dada a complexidade de parâmetros que interagem entre si no produto proposto.

A produção assistida por computador como parte do processo de projeto tornou-se um componente intrínseco do projeto computacional, dando acesso a um sistema de produção capaz de integrar as necessidades de projeto e fabricação em diversas escalas (Sass, 2010). O projeto tridimensional em conjunto com a manufatura tridimensional possibilita análises das relações que ocorrem entre a fisicalidade e o comportamento do produto desenvolvido, possibilitando a comunicação entre o real e o virtual (Romero, 2018). Assim, o design do produto se dará por intermédio de tecnologias de Fabricação Digital em processos file-to-factory, o qual tem como objetivo explorar, desenvolver e implementar modelos baseados nos parâmetros estabelecidos pelo projeto, lidos como informações numéricas. Para a geração de formas complexas, serão usados sistemas CAD [Computer Aided Design], integradamente com a geração de análises performáticas por meio do $\mathrm{CAE}$ [Computer Aided Engineering], por fim, a fabricação por meio do CAM [Computer Aided Manufacturing].

O material tem como função proporcionar funcionalidade e personalidade ao produto e a escolha de um material não pode ser feita independentemente da escolha do processo pelo qual o material é originado, elaborado, acabado e tratado (Ashby, 1995). A prototipagem rápida tridimensional está diretamente vinculada ao material que será impresso o produto e tais impressoras apresentam uma gama limitada de materiais para impressão. Não diferente disso, a área útil de impressão das impressoras 3D é limitada, implicando em tomadas de decisão de projeto. Material e tipo de impressora são alguns dos inputs decisivos no desenvolvimento do projeto.

\section{EXPERIMENTOS PREVISTOS}

Os experimentos relacionados aos vestíveis tecnológicos se dividem em etapas que se relacionam constantemente. A primeira etapa com ênfase técnica e de desenvolvimento de projeto, ocorrem 
simultaneamente, uma vez que o desenvolvimento do design do produto está diretamente vinculado às tecnologias computacionais, as quais neste caso fazem parte do processo de criação. Por se tratar de um processo interdisciplinar, questões sobre eletrônica, aspectos clínicos e design de produto se interpolam, promovendo ajustes e revisões constantes. Na ênfase técnica são testadas diversas configurações dos elementos externos ao sistema como: 1. Tipos de scanners 3D - determinou-se que o escaneamento do braço do paciente deve ser realizado no laboratório, já que os pacientes apresentam grande dificuldade de locomoção e transporte, além disso, o modo como serão escaneados seus membros superiores, pois devido a sua imobilidade, os perímetros de braços e antebraços se alteram quando apoiados em um plano em relação à quando estão elevados. Para se determinar o nível de precisão do aparelho em relação à necessidade para o desenvolvimento do produto, adotou-se o membro superior de um indivíduo saudável a ser escaneado (Figura 11). A princípio o scanner portátil utilizado, 3D Isense (3D Systems, Rock Hill, South Carolina, USA), atende o objetivo desejado no quesito precisão. 2. O tipo de impressora 3D - duas tecnologias de impressão estão sendo avaliadas e testadas no momento, de acordo com a sua disponibilidade de uso e verificação de possibilidades de comportamento do material em relação à sua maleabilidade e performance. A primeira é a impressão por meio da tecnologia de deposição de camadas, conhecida como tecnologia FDM - Fused Deposition Modeling e o material a ser testado é o filamento flexível de poliéster e a segunda é a tecnologia de impressão por sinterização de pó a laser, conhecido como tecnologia SLS- Sinterização Seletiva a Laser e o material é um polímero em pó, ainda não definido. 3 . Bateria que alimentará todo o sistema. 4. Estimulador Elétrico Neuromuscular. 5. Equipamento de captura de imagem de movimento, pelo Sistema Optoeletrônico de Captura Tridimensional, para avaliação de grandezas cinemáticas (velocidade e aceleração) e suavidade dos movimentos dos pacientes com o uso do vestível e 6 . 0 conjunto de software que irão compor o desenvolvimento do vestível, uma vez que eles devam se comunicar. No momento tem-se usado o Skanect, para o escaneamento do braço, o MeshLab, para reparos do modelo 3D resultante do escaneamento do membro superior, o Rhino3D em conjunto com o plugin Grasshopper para a modelagem tridimensional de algoritmo generativo.

Na ênfase de desenvolvimento de produto são discutidos os parâmetros que devem compor o sistema em conjunto com a geração das ideias criativas relacionadas ao seu design, por meio de esboços e explorações projetuais via software de modelagem 3D. Os parâmetros discutidos são os depoimentos e a participação dos pacientes e equipe técnica de como melhorar o sistema, o protocolo de funcionamento do sistema de reabilitação, a composição dos componentes de eletrônica com o membro do paciente, tendo a participação conjunta da equipe clínica, pacientes, designers e engenheiros, o tipo de material a ser usado de modo que torne o vestível ergonômico e maleável (esta parte da etapa está em andamento). Inicialmente serão desenvolvidos dois sistemas tridimensionais, sendo que o primeiro corresponderá ao vestível do paciente 1 com inputs fixos e variáveis (dimensões perimetrais dos membros superiores), o segundo corresponderá ao vestível do paciente 2, em que os inputs fixos do vestível do paciente 1 permanecerão, com a alteração dos inputs variáveis (dimensões do membro superior) referentes ao paciente 2. Estas simulações servirão para verificar se o primeiro sistema aninhará o segundo de modo favorável. Além disso, modelos deverão ser produzidos para ajustes e possíveis redesenhos. A cada conclusão de ciclo o sistema será retroalimentado e revisitado para a correção de possíveis erros e aprimoramento do projeto. A retroalimentação faz com que dados de saída retornem ao sistema e passem a ser dados de entrada e, numa complexidade maior, um sistema passa a aninhar outro sistema, num processo de complexas inter-relações, caracterizando-os como sistemas não lineares (Dubberly, 2007). Quando processos de projeto são desenvolvidos em ciclos de retroalimentação, levando à uma mudança maior, as recursividades resultantes podem abrir possibilidades de observação para algo inovador.

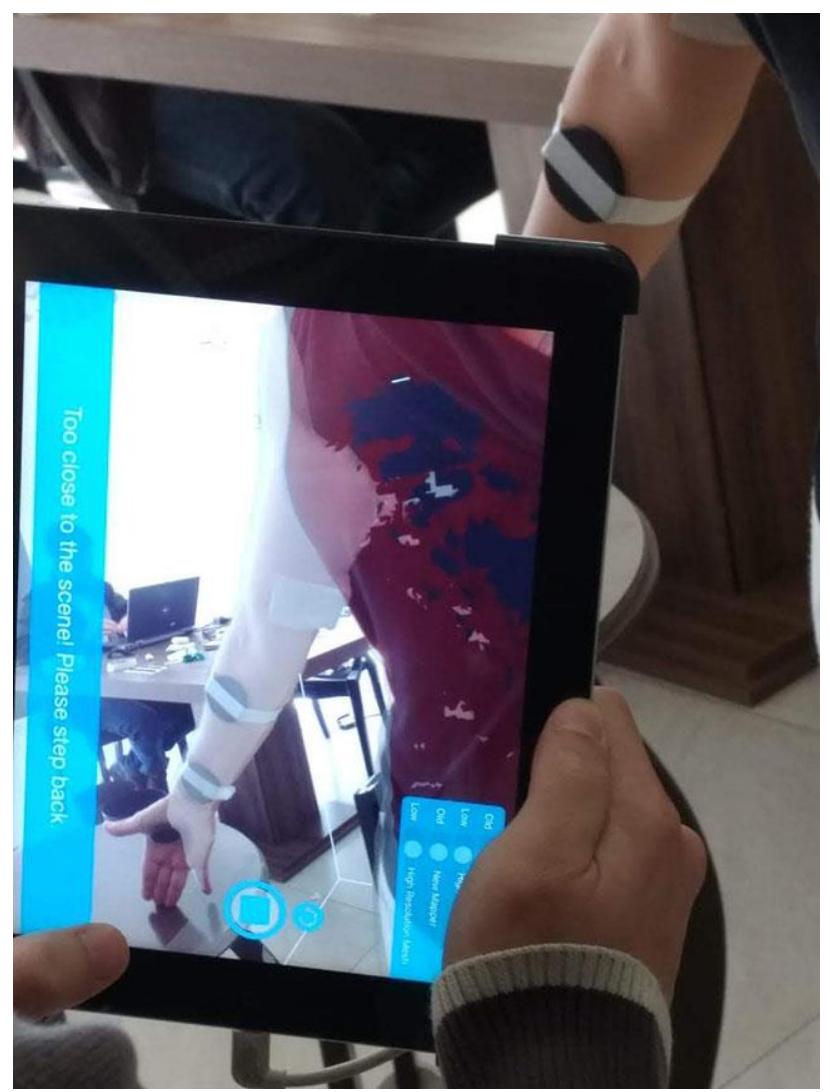

Figura 11. Scanning 3D de indivíduo saudável. Sacnner portátil 3D Isense. Fonte: Autoria própria, 2018

A segunda etapa refere-se à produção de experimentos. Num primeiro momento, um vestível será produzido em impressora 3D, testado pelo paciente e equipe técnica quanto a sua colocação. Também serão verificados se os eletrodos, incorporados ao vestível, estão funcionando corretamente, durante o procedimento da reabilitação e, por fim, o produto será avaliado de quatro maneiras: 1. Pela equipe técnica e pacientes quanto a sua funcionalidade, ergonomia e eficiência, 2. Por meio de equipamento com sistema de captura de imagem de movimento para avaliar a suavidade dos movimentos, 3 . Por meio de sensores de estimulação tátil para detecção 
de força e 4. Os dados resultantes do vestível serão analisados e comparados aos dados do sistema atual.

Após ajustes e aprovado o primeiro vestível serão dados andamentos aos ajustes do segundo vestível, com base no primeiro e que atenderá 0 segundo paciente voluntário. A ordem da produção dos vestíveis depende da disponibilidade dos voluntários para o escaneamento dos seus membros superiores. $O$ fator que determina 0 desenvolvimento de 5 vestíveis é devido ao fato dos experimentos atenderem uma gama variada de perfis e saber se os vestíveis apresentam alguma consideração em relação ao gênero, idade e perfil do paciente.

\section{RESULTADOS}

Muitos direcionamentos podem resultar dos experimentos acima propostos. Entende-se que estes encaminhamentos devam tentar responder às seguintes questões: O primeiro sistema desenvolvido tem a capacidade de aninhar o segundo sistema? Quais são as possibilidades não previstas que o sistema desenvolvido proporcionou? O sistema poderia ser controlado à distância por um profissional da saúde autorizado, uma vez que a parametrização visa a precisão e eficiência do sistema, tornando-o portátil? Quais seriam as possíveis formas de interação do vestível com o usuário de modo que ele sirva de plataforma de comunicação? $O$ que norteia este trabalho é a relação do universo de programação determinística do sistema proposto e as possíveis alternativas imprevisíveis que ele pode trazer.

\section{DISCUSSÃO}

Assim, esta pesquisa impacta diretamente na eficiência do processo de reabilitação de pacientes tetraplégicos, na melhora das suas condições de saúde dando abertura para novas possibilidades de uso e possível reinserção social, bem como avanços das técnicas de reabilitação de membros superiores em lesados medulares. Contribui com a exploração de possibilidades projetuais digitais com o uso de plug-in de otimização de código aberto e com o registro de dados informacionais de todo o processo, gerando deste modo, discussões na área de design de produto e em áreas afins que possam utilizar o mesmo processo.

\section{CONTRIBUIÇÃO}

Este projeto interdisciplinar tem como objetivo caminhar em conjunto com o desenvolvimento das tecnologias do LABCIBER e LABRAL.

O estágio em que as pesquisas se encontram nestes laboratórios requer um avanço na interface desta tecnologia com o paciente, para que além de serem beneficiados por toda ciência envolvida (Design, Bioengenharia, Medicina, Computação e Engenharia), possam utilizar deste enorme benefício cibernético, de forma mais próxima da realidade humana.

\section{AGRADECIMENTOS}

O presente trabalho foi realizado com apoio da Coordenação de Aperfeiçoamento de Pessoal de Nível Superior - Brasil (CAPES) - Código de Financiamento 001. Agradecemos também à FAPESP e ao CNPq.

\section{REFERÊNCIAS}

Alonso, K. C. (2015). Estimulação elétrica neuromuscular associada a movimentos funcionais e repetitivos: avaliação da efetividade desta abordagem terapêutica desenvolvida para reabilitação dos membros superiores de indivíduos com tetraplegia. Tese (doutorado em Fisiopatologia Cirúrgica) Faculdade de Ciências Médicas, Universidade Estadual de Campinas. Campinas, p. 113.

Ashby, M.; Jonson; K. (2011). Materiais e design: arte e ciência da seleção de materiais no design de produto. Rio de Janeiro: Elsevier.

Dubberly, H., Pangaro, P. (2007). Cybernetics and Service-Craft: Language for Behavior-Focused Design. Kybernetes Emerald Group Publishing Limited, Southsea, v. 36, p. 13011317

Emiris, I., Fisikopoulos, V. (2009). Voronoi diagram and Delaunay triangulation. Dept. of Informatics \& Telecommunications, University of Athens. Retrieved from: <https://eclass.uoa.gr/modules/document/file.php/D42/\%CE $\% 94 \%$ CE\%B9\%CE\%B1\%CF\%86\%CE\%AC\%CE\%BD\%CE $\%$ B5\%CE\%B9\%CE\%B5\%CF\%82/2a.delaunay.pdf>

Eng, J. J.; Miller, W. C. (2006). Rehabilitation: from bedside to community following spinal cord injury (SCI). In: Eng, J. J.; Teasell, R. W.; Miller, W. C.; Wolfe, D. L.; Townson, A. F.; Aubut, J.; Abramson, C.; Hsieh, J. T. C.; Connolly, S. Spinal cord injury rehabilitation evidence. Vancouver. cap. 1, p. 1629.

GALVÃO, C. L. (2003). Dimensionamento de sistemas de distribuição através do diagrama multiplicativo de Voronoi com pesos. Tese (doutorado em Transportes e Logística) Faculdade de Engenharia de Produção e Sistemas, Universidade Federal de Santa Catarina. Florianópolis.

Ho, C. H., Triolo, R. J., Elias, A. L., Kilgore, K. L., DiMarco, A. F., Bogie, K., Mushahwar, V. K. (2014). Functional Electrical Stimulation and Spinal Cord Injury. Physical Medicine and Rehabilitation Clinics of North America, 25(3), 631-ix. http://doi.org/10.1016/j.pmr.2014.05.001ry

Malešević, N. M. Lana Z Popović Maneski, P. Z. L., Ilić, V., Jorgovanović, N., Bijelić, G., Keller, T., Popović, B. D. (2012). A multi-pad electrode based functional electrical stimulation system for restoration of grasp. Journal of NeuroEngineering and Rehabilitation. https://doi.org/10.1186/1743-0003-9-66

Oxman, N. (2010). Material-based design computation. Thesis (Doctor of Philosophy in Architecture: Design and Computation) - Department of Architecture. Massachusetts Institute of Technology,

Parente, N. L. (2017). Controlador de estimulador neuromuscular utilizando Raspberry Pi. Monografia (Graduação em Engenharia Elétrica com ênfase em Eletrônica), Escola de Engenharia de Snão Carlos da Universidade de São Paulo.

Peckham, P. H., Keith, M. W., Freehafer, A. A. (1988). Restoration of functional control by electrical stimulation in the upper extremity of the quadriplegic patient. Journal Bone and Joint Surgery 70-A (1): 144-148.

Romero, M., Perego, P., Andreoni, G., Costa, F. (2010). New Strategies for Technology Products Development in Health Care. Doi: 10.5772/10415.

Romero, M. (2018). Interaction and Forming, How Industrial Design Is Changing. In M. Rossi, \& G. Buratti (Eds) Computational Morphologies (pp. 147-156). Switzerland: Springer, Cham,

Sass, L. (2010). The Next Revolution: Digital Buildings Kit. In New Frontiers: Proceedings of the 15th International Conference on Computer Aided Architectural Design Research in Asia, 545-553. Hong Kong: CAADRIA. Retrieved from http://papers.cumincad.org/cgibin/works/paper/caadria2010_099

Snoek, G. J., IJzerman, M. J., In't Groen, Fa. C. G., Stoffers, T. S., and Zilvold, G. (2000). Use of the NESS Handmaster to 
restore handfunction in tetraplegia: Clinical experiences in ten patients. Spinal Cord, 38, 244. doi: 10.1038/sj.sc.3100980

Studer, R., Benjamins, V. R., Fensel, D. (1998) Knowledge Engineering: Principles and Methods. Data \& Knowledge Engineering, v. 25, n. 1-2, p. 161-197. doi: 10.1016/S0169023X(97)00056-6

Varoto, R., Cliquet Jr, A. (2015). Experiencing Functional Electrical Stimulation Roots on Education, and Clinical Developments in Paraplegia and Tetraplegia With
Technological Innovation. Artificial Organs, v. 39, p. n/a-n/a. doi: 10.1111/aor.12620

Varoto, R., Ferreira, V. M. V., Alonso, K. C.; BEINOTTI, F., Cliquet Jr, A. (2011). Hybrid System: A Pilot Evaluation for a Therapeutic Proposal in Quadriplegia. In: 16th Annual International FES Society Conference, 2011, São Paulo. 16th IFESS Annual Conference, 2011. São Paulo: Atha Comunicação e Editora.

Woodbury, R. F. (2010). Elements of parametric design. London: Routledge. 